\title{
Double Orifice Fissured Subaortic Membrane in the Adult
}

\author{
Giordano Zampi ${ }^{1}$, Marzia Cottini ${ }^{2,}{ }^{,}$, Amedeo Pergolini ${ }^{2}$, \\ Vincenzo Polizzi ${ }^{2}$, Francesco Musumeci ${ }^{2}$ \\ ${ }^{1}$ MD, U.O.C. Cardiologia ed Emodinamica Ospedale Belcolle, Viterbo, Italy \\ ${ }^{2} \mathrm{MD}$, Department of Heart and Vessels, Cardiac Surgery Unit and Heart Trans- \\ plantation Center, S. Camillo-Forlanini Hospital, Rome, Italy \\ * Corresponding author: Marzia Cottini, MD, Department of Heart and Vessels, \\ Cardiac Surgery Unit and Heart Transplantation Center, S. Camillo-Forlanini \\ Hospital, Rome, Italy.E-mail: marzia.cottini@hotmail.it
}

DOI: $10.21859 / \mathrm{ijcp}-020107$

Submited: 13.12 .2016

Accepted: 23.01 .2016

\section{Keywords:}

Aortic Stenosis

Subvalvular

Echocardiography

Three-Dimensional

Heart Defects

Congenital

(C) 2016. International Journal of

Cardiovascular Practice.

\begin{abstract}
Left ventricle outflow tract obstruction is a relatively common form of congenital heart disease, occurring in 2.8 out of 10,000 live births and accounts for 3-6\% of congenital heart diseases. Subvalvular aortic stenosis can be either a fixed stenosis resulting from subaortic membrane or a dynamic stenosis because of hypertrophic cardiomyopathy. We described an original and rare image of double orifice fissured subaortic membrane in the adult.
\end{abstract}

\section{INTRODUCTION}

Left ventricular outflow tract obstructions (LVOTOs) encompass a series of stenotic lesions starting in the anatomic left ventricular outflow tract (LVOT) and stretching to the descending portion of the aortic arch. Obstruction may be subvalvular, valvular, or supravalvular [1-4]. Fixed subaortic stenosis may be due to a discrete fibrous membrane, a muscular narrowing, or a combination of them. The obstruction may be focal, as in a discrete membrane, or more diffused, resulting in a tunnel leading out of the left ventricle (Table 1).

The fibromuscular fixed subaortic stenosis is the most frequent, and in many cases the tunnel-type lesions are associated with a greater degree of stenosis. They are isolated or acquired and their prevalence in adults with congenital heart disease is $6.5 \%$, with a male to female ratio of $2: 1$ [4-7]. We reported an interesting and reliable case of double orifice fixed subaortic membrane in adults.

\section{CASE PRESENTATION}

A 55-year-old Caucasian female with a 6-month clinical history of breathlessness and mild fatigue referred for presumptive aortic stenosis to our EchoLab. The first transthoracic echocardiogram (TTE) revealed a thickened aortic cusp with a mild stenosis (Effective Orifice Area [EOA] $1.7 \mathrm{cmq}$, Mean Aortic Valve Pressure Gradient [MAVPG] $20 \mathrm{mmHg}$, Doppler

\begin{tabular}{l} 
Table 1: The Most Important Characteristics of the Sub- \\
aortic Stenosis \\
Types \\
Acquired \\
Congenital \\
Familiar \\
Pathogenesis \\
Fibrous membrane \\
Muscular Narrowing \\
Combination of them \\
Presentation \\
Isolated \\
Combined \\
Bicuspid Valve \\
Shone's complex \\
Ventricular Septal Defect \\
Ankylosing Spondilitis $(8)$ \\
\hline
\end{tabular}

Velocity Index [DVI] 0.57) and a mild regurgitation. A physical examination documented a $3 / 6$ systolic ejection murmur and no basilar rales; the blood pressure was $130 / 80 \mathrm{mmHg}$. The transesophageal echocardiography (TEE) revealed a normal aortic morphology (tricuspid, Fig 1a), but high 


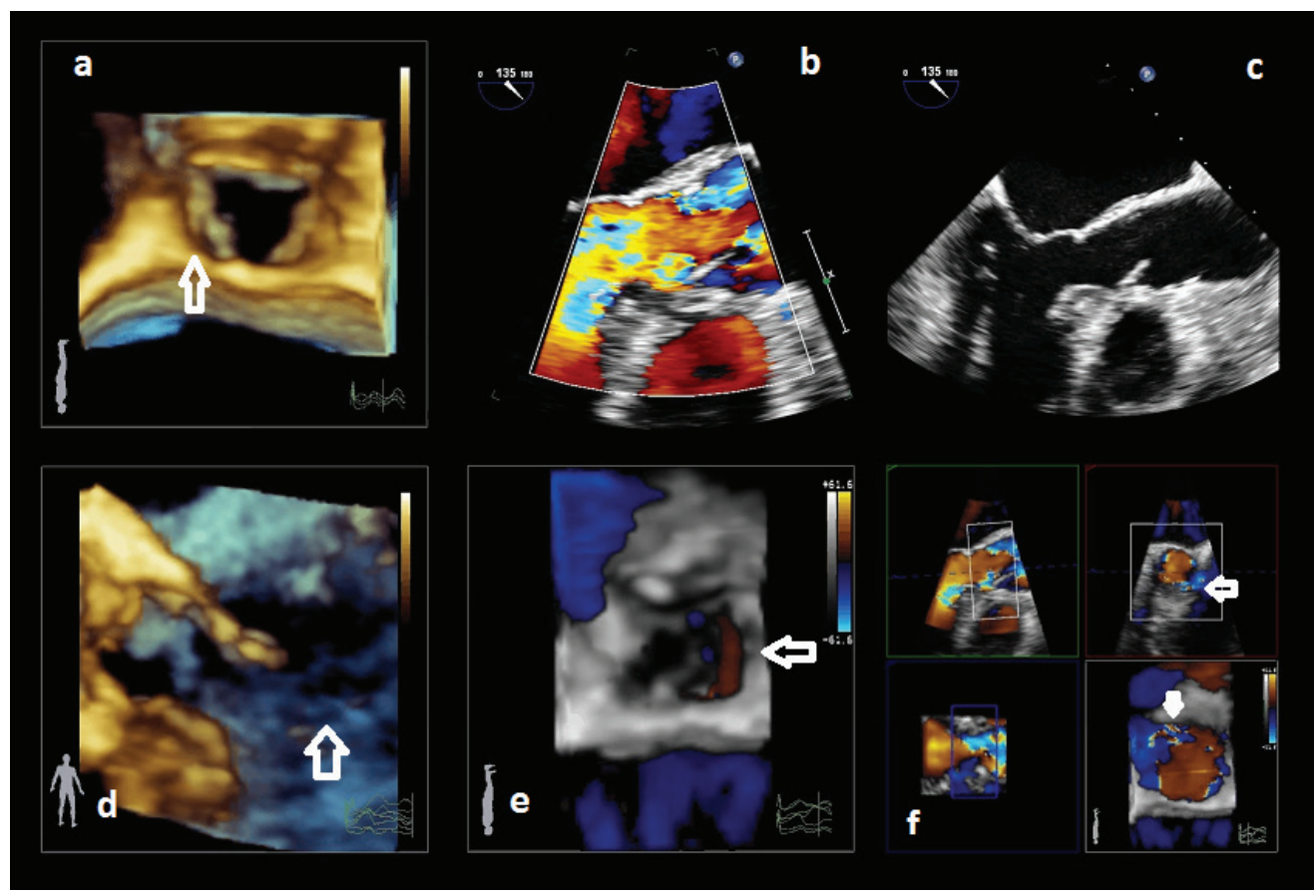

Figure 1: Ttransesophageal echocardiography (a) revealing the normal tricuspid morphology and the high systolic flow velocity in the left ventricular outflow tract (LVOT, whit arrow) a sign of vascular turbulence due to a suspicious and linear structure detected in the LVOT (c, arrow). The three-dimensional TEE highlighted the spatial assessment of the sub-aortic membrane (d, arrow), in particularly the sub-aortic membrane was fissured in its upper portion (e) and the color Doppler documented the flow coming through fenestrations (f, double orifice).

systolic flow velocity in the left ventricular outflow tract (LVOT) with documented evidence of turbulence on colour-Doppler images (Fig 1b).

Due to progressive diagnostic examination, a suspicious and linear structure could be detected in the LVOT, about $5 \mathrm{~mm}$ below the aortic valve: a sub-aortic membrane (Fig 1c, arrow). Live three-dimensional TEE (3D-TEE, using a fully sampler 3D transducer, Philips IE 33, Handover) improved the spatial assessment of the sub-aortic membrane (Fig 1d). On the other hand, using multiplanar review on Xcelera workstation with the use of QLab software (Philips Medical Systems), the sub-aortic membrane was better analyzed: it was fissured in its upper portion (Fig 1e) and the color Doppler documented the flow coming through fenestrations (double orifice, Fig 1f). Videos are available as online supplements. The patient was enrolled for yearly follow-up echocardiography and treated with low dose beta-blocker (Bisoprolol).

\section{DISCUSSION}

Subaortic stenosis carries considerable morbidity and mortality and some of these patients could progress to develop severe stenosis and aortic regurgitation [8]. Patients with subaortic stenosis could present no signs or symptoms of chest pain, syncope and congestive heart failure depending of the severity of obstruction. According to Ayoub et al. [9], misdiagnosis and failure to recognize fixed left ventricular outflow tract lesions (such as subvalvolar aortic stenosis due to membrane) may result in morbidity as a result of inappropriate therapy and delay of definitive surgical treatment. Hence, it is necessary to identify the correct type and level of obstruction in the LVOT by careful correlation of clinical examination, by Doppler evaluation, and advanced imaging findings. The 2-D Echocardiogram is the primary diagnostic method for diagnosing subaortic stenosis and distinguishing a discrete fibrous membrane from diffuse fibromuscular type of obstruction. The 3-D Echocardiogram could be the gold standard for the detection of LVOT obstruction and their differential diagnosis because of the accuracy, specificity of the images, and the definition of the cardiac details. The 3-D view of the heart addresses a better comprehension of the anatomical and functional LVOT obstruction in order to program the best therapeutic choice. Computed tomography could help to collect further information of the hemodynamic details of obstruction and its relationships with the surrounding anatomical structures. Furthermore, cardiac MRI is a reliable and useful support to identify the type of LVOT obstruction and cardiac chambers characteristics. As a supplement to them, if the diagnosis was still doubtful, we suggest intra-cardiac echocardiogram (ICE) [10].

According to ESC Guidelines for the management of grownup congenital heart disease (2010) [11], the therapeutic choice of subaortic stenosis could depend to the degree of severity, mean gradient, and continuity equation-calculated effective orifice area (AVA). Medical therapy could be for inoperable patients and to control heart failure. Surgery is required in symptomatic patients; in selected adolescents and young adults with non-calcified valves, balloon valvuloplasty may be considered. In patients with calcified valves, the treatment of choice is valve replacement. The Ross procedure has been suggested for patients of childbearing age and for those who want to avoid anticoagulation. Percutaneous valve implantation has become an alternative technique for treating stenosis of the pulmonary valve substitute (homo- 
graft). Transcatheter aortic valve implantation currently has no place in the treatment of congenital AS.

In our case, the stenosis was moderate and the patient referred with breathlessness and mild fatigue, no ascending aortic dilatation, and no left ventricle dysfunction; hence, we successfully treated the patient with medical therapy and periodical control by echocardiogram in our ambulatory. The obstruction of LVOT, in particular subaortic membrane stenosis, is a rare and misdiagnosed disease, not to underestimate in adults.

\section{Human Rights Statements and Informed Consent}

All procedures followed were in accordance with the ethical standards of the responsible committee on human experimentation (institutional and national) and with the Helsinki Declaration of 1964 and later revisions. Informed consent was obtained from the patient for being included in the image report.

\section{FUNDING}

Authors declared there is no funding or support.

\section{REFERENCE}

1. Ferencz C, Neill C. Cardiovascular malformations: prevalence at livebirth. Neonatal heart disease. New York: Springer; 1992. p. 19-29.

2. Brown JW, Ruzmetov M, Vijay P, Rodefeld MD, Turrentine MW Surgery for aortic stenosis in children: a 40-year experience. Ann
Thorac Surg. 2003;76(5):1398-411. PMID: 14602258

3. Oliver JM, Gonzalez A, Gallego P, Sanchez-Recalde A, Benito F, Mesa JM. Discrete subaortic stenosis in adults: increased prevalence and slow rate of progression of the obstruction and aortic regurgitation. J Am Coll Cardiol. 2001;38(3):835-42. PMID: 11527642

4. Aboulhosn J, Child JS. Left ventricular outflow obstruction: subaortic stenosis, bicuspid aortic valve, supravalvar aortic stenosis, and coarctation of the aorta. Circulation. 2006;114(22):2412-22. DOI 10.1161/CIRCULATIONAHA.105.592089 PMID: 17130357

5. Ezon DS. Fixed subaortic stenosis: a clinical dilemma for clinicians and patients. Congenit Heart Dis. 2013;8(5):450-6. DOI: 10.1111 chd.12127 PMID: 23947905

6. Barekatain A, Fanari Z, Hammami S, Qureshi W. Subvalvular Aortic Stenosis. Del Med J. 2015;87(11):346-8. PMID: 26731888

7. Valeske K, Huber C, Mueller M, Boning A, Hijjeh N, Schranz D, et al. The dilemma of subaortic stenosis--a single center experience of 15 years with a review of the literature. Thorac Cardiovasc Surg. 2011;59(5):293-7. DOI: 10.1055/s-0030-1271039 PMID: $\underline{21544788}$

8. Yacoub M, Onuzo O, Riedel B, Radley-Smith R. Mobilization of the left and right fibrous trigones for relief of severe left ventricular outflow obstruction. J Thorac Cardiovasc Surg. 1999;117(1):126-32; discussion 32-3. PMID: 9869766

9. Ayoub C, Brieger D, Chard R, Yiannikas J. Fixed left ventricular outflow tract obstruction mimicking hypertrophic obstructive cardiomyopathy: pitfalls in diagnosis. Echocardiography. 2016;33(11):175361. DOI: $10.1111 /$ echo.13356 PMID: 27613242

10. Sgueglia GA, Palombaro G, Pucci E. [Intracardiac echography in in terventional cardiology]. G Ital Cardiol (Rome). 2013;14(10):6508. DOI: $10.1714 / 1335.14831$ PMID: 24121889

11. Baumgartner H, Bonhoeffer P, De Groot NM, de Haan F, Deanfield JE, Galie N, et al. ESC Guidelines for the management of grown-up congenital heart disease (new version 2010). Eur Heart J. 2010;31(23):2915-57. DOI: 10.1093/eurheartj/ehq249 PMID: $\underline{20801927}$ 